\title{
Hallermann Syndrome
}

National Cancer Institute

\section{Source}

National Cancer Institute. Hallermann Syndrome. NCI Thesaurus. Code C84746.

A very rare syndrome characterized by multiple congenital abnormalities including abnormally shaped head, mandibular hypoplasia, parrot nose, bilateral congenital cataracts, microphthalmia, dwarfism and hypotrichosis. 